\title{
ACMG's continuing medical education program: A new initiative
}

The continuum of medical education is not immune to the turbulent changes sweeping through our health care systems. While providers, consumers, policy-makers, and insurers grapple with the economic and political forces driving the changes in health care, medical educators have begun to initiate major reforms in undergraduate and graduate medical education as well as in continuing medical education (CME).

Indeed many of the changes in medical education mirror the emerging national trends and priorities in health care. They are designed to address a general growing concern about the need to ensure the delivery of competent and consistent quality care. For example many medical school curricula now include problem-based learning, medical ethics, an emphasis on the development of critical analytic skills, interdisciplinary approaches to care, and community-based learning experiences.

In addition to strengthening their trainees' communitybased clinical rotations, many graduate medical education programs now promote a generalist approach. These changes directly reflect the national shift to train more primary care providers and fewer specialists.

New approaches, concepts, and ideas to improve existing CME methodologies are also being refined. Long recognized as a major facilitator of change in practitioner behavior, CME is regarded as an important means for physicians to update the knowledge and skills necessary to provide the best possible health care to their patients. ${ }^{1}$

\section{What is CME?}

According to the Accreditation Council for Continuing Medical Education (ACCME), "CME consists of educational activities which serve to maintain, develop or increase the knowledge, skills and professional performance and relationships that a physician uses to provide services for patients, the public or the profession. The content of CME is that body of knowledge and skills generally recognized and accepted by the profession as within the basic medical sciences, the discipline of clinical medicine, and the provision of health care to the public." ${ }^{2}$

This broad definition of CME recognizes that all continuing educational activities that assist physicians in carrying out their professional responsibilities more effectively and efficiently are CME. Continuing educational activities that respond to a physicians' nonprofessional educational needs or interests are not $\mathrm{CME}^{2}$

Over the years, concerns surfaced about the effectiveness and efficiency of CME. ${ }^{1} \mathrm{CME}$ was viewed as episodic and lacking in cohesiveness. Data about the effectiveness of educational strategies designed to change physician behavior and health care outcomes were sparse.
A recent review of the ever-expanding literature on CME yielded several papers that provide reassurance that CME really works. ${ }^{3,4}$ However at the same time these papers revealed that there are important steps that must be taken to enhance the effectiveness of CME activities.

These include careful planning, the implementation of targeted ongoing needs assessment, the meticulous preparation of a "focused stream of formal and informal educational activities the content of which is based on an understanding of what knowledge, skills and attitudes the learners need to improve their performance so that patients receive the very best possible care." A strong evaluation component must be part of the structural framework of each activity. Traditionally such evaluation schemes relied on the assessment of participation, statements about the level of satisfaction and measures of changes in knowledge, skills, or attitudes of the participants. Today more complete evaluation plans should include assessment of improved performance and measures of patient, societal, or economic outcomes.

In 1995 Davis et al. ${ }^{4}$ published a pivotal paper entitled, "A Systematic Review of the Effect of CME Strategies," which supports the application of scientific and theoretical principles to evaluate changes in health professionals' performances in a move toward "evidence-based CME." In their quest for effective interventions, the authors identified practice-enabling strategies, including reminders, reinforcing strategies, the inclusion of recognized opinion leaders throughout the process, patient mediated strategies, role-playing, and peer discussion.

CME interventions can alter physicians performance. The rate of change will vary from practitioner to practitioner. Motivation differences, existing levels of knowledge, and the willingness to recognize gaps of knowledge account for some of these discrepancies. There is also a need to recognize barriers to change and to devise strategies to overcome these barriers to effect change. ${ }^{4}$

Changes in physicians' performance should have an impact on health care outcomes. Yet the alterations in health care outcomes are generally small. The lag may be due to the limited number and effectiveness of the proposed clinical intervention as well as the reluctance of patients to buy into physician recommendations. ${ }^{4}$

All of these issues will be important to genetic health care professionals as they attempt to devise mechanisms to effect appropriate educational linkages with other professional groups particularly in their efforts to increase the genetic literacy of nongenetic health care providers. They will also play a major role in the development of our profession's strategies to achieve recertification. 
What is the American College of Medical Genetics' approach to $C M E$ ?

The American College of Medical Genetics (ACMG) CME Program fits into the overall mission of ACMG, which is to provide education, resources, and a voice for the medical genetic profession. To fulfill its mission, the ACMG strives to advance the art and science of medical genetics by maintenance of high standards in education, practice, and research.

Established in 1991, the ACMG is a young organization. Yet ACMG's fledgling CME Program has received provisional accreditation by the ACCME. College members have worked hard to enhance and refine all aspects of ACMG's CME Program. To this end, the design of ACMG's 1999 Annual Meeting was modified to encourage more interaction through the use of focus groups. ACMG also offered its first review course in medical genetics this spring.

This issue of Genetics in Medicine includes another firstthe opportunity for journal subscribers to engage in a new CME educational self-assessment examination and evaluation program. As part of this new effort, we plan to offer this CME activity as a regular journal feature. This ongoing activity will enable us to cover a wide range of topics on all aspects of medical genetics. It will easily be incorporated into the electronic version of our journal.

We invite all of our readers to actively participate in this activity as well as give our editor feedback about this section. Guidelines for interested authors will be circulated. We also invite our members to describe their research findings related to CME.

\section{References}

1. Moore DE. Needs assessment in the new health care environment: Combining discrepancy analysis and outcomes to create more effective CME. JCEHP 1998;18:133-141.

2. Essentials, and Guidelines for Accreditation of Sponsors of Continuing Medical Education, and Standards for Commercial Support, and Enduring Materials. Accreditation Council for Continuing Medical Education. Chicago IL, 1987.

3. Fox RD, Mazmanian PE, Putnam RW. Changing and Learning in the Lives of Physicians. New York: Praeger, 1989.

4. Davis DA, Thomson MA, Oxman AD, Haynes RB. Changing physician performance. A systematic review of the effect of continuing medical education strategies. JAMA $1995 ; 274: 700-705$.
Jessica G. Davis, MD, FACMG

New York Presbyterian Hospital Division of Human Genetics 